\title{
Reconstruction of a large chest wall defect using bilateral pectoralis major myocutaneous flaps and V-Y rotation advancement flaps: a case report
}

\author{
Gang Yeon Jo ${ }^{1}$, Jin Myung Yoon ${ }^{1}$, Sae Hwi Ki ${ }^{1,2}$ \\ ${ }^{1}$ Department of Plastic and Reconstructive Surgery, Inha University Hospital, Incheon; ${ }^{2}$ Department of Plastic and Reconstructive Surgery, \\ School of Medicine, Inha University, Incheon, Korea
}

\begin{abstract}
Bilateral pectoralis major myocutaneous (PMMC) flaps are commonly used to reconstruct large chest wall defects. We report a case of large chest wall defect reconstruction using bilateral PMMC flaps augmented with axillary V-Y advancement rotation flaps for additional flap advancement. A 74-year-old male patient was operated on for recurrent glottic squamous cell carcinoma. Excision of the tumor resulted in a $10 \times 10 \mathrm{~cm}$ defect in the anterior chest wall. Bilateral PMMC flaps were raised to cover the chest wall defect. For further flap advancement, $\mathrm{V}-\mathrm{Y}$ rotation advancement flaps from both axillae were added to allow complete closure. All flaps survived completely, and postoperative shoulder abduction was not limited $\left(100^{\circ}\right.$ on the right side and $92^{\circ}$ on the left). Age-related skin redundancy in the axillae enabled the use of $\mathrm{V}-\mathrm{Y}$ rotation advancement flaps without limitation of shoulder motion. Bilateral PMMC advancement flaps and the additional use of V-Y rotation advancement flaps from both axillae may be a useful reconstructive option for very large chest wall defects in older patients.
\end{abstract}

Keywords Pectoralis muscle / Thoracic wall / Neoplasms / Case reports

Received: July 16, 2021 - Revised: November 4, 2021 • Accepted: December 1, 2021

pISSN: 2234-6163 • elSSN: 2234-6171 • https://doi.org/10.5999/aps.2021.01368• Arch Plast Surg 2022;49:39-42

\author{
Correspondence: Sae Hwi Ki \\ Department of Plastic and \\ Reconstructive Surgery, School of \\ Medicine, Inha University, \\ 27 Inhang-ro, Jung-gu, Incheon \\ 22332, Korea \\ Tel: +82-32-890-3619 \\ Fax: +82-32-890-2918 \\ E-mail:mdkee0923@gmail.com
}

\section{INTRODUCTION}

Chest wall defects are commonly caused by trauma, deep sternal wound infections, chronic empyema, congenital defects, and tumor resection [1]. Chest wall reconstruction can be divided into skeletal and soft tissue reconstructions. Skeletal support to prevent flail chest and subsequent respiratory failure is an important requirement for the successful management of chest wall tumors [1-4]. For reconstruction of the central area, a unilateral pectoralis major myocutaneous (PMMC) flap can be used for small defects, and bilateral PMMC flaps, bilateral pectoralis major turn-over flaps, a rectus abdominis muscle flap, a free flap, or other options can be used for large defects [2-4]. Here, we describe a case of successful reconstruction using bilateral PMMC flaps and V-Y rotation advancement flaps (for further skin advancement) in an elderly patient with a large defect $(10 \times 10 \mathrm{~cm})$ in the center of his chest wall.

\section{CASE}

A 74-year-old man with a 30-pack-year smoking history with squamous cell carcinoma (SCC) of the glottis, underwent total laryngectomy and modified radical neck dissection 7 months before presentation to our otolaryngology department. Four 


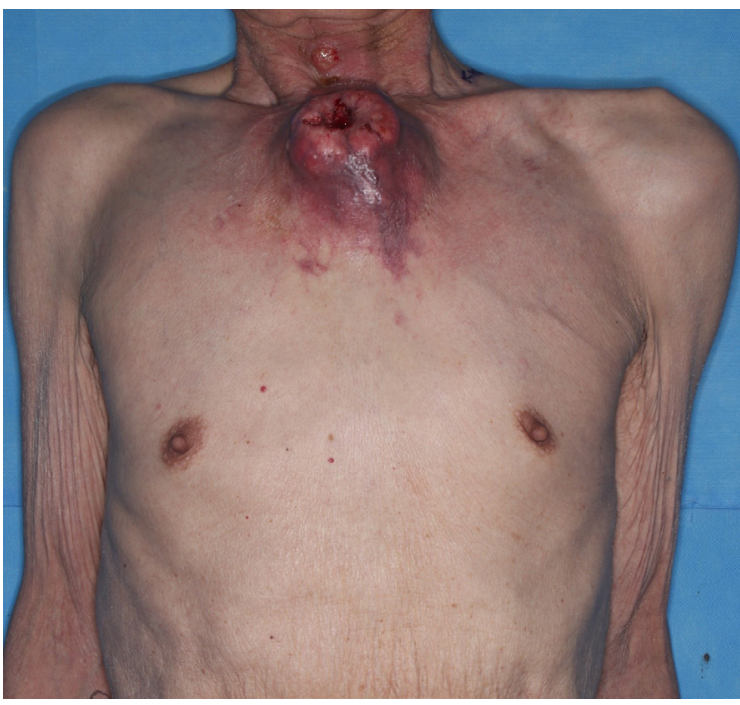

Fig. 1. Preoperative photograph. Recurrent cancer at the previous tracheostomy site.

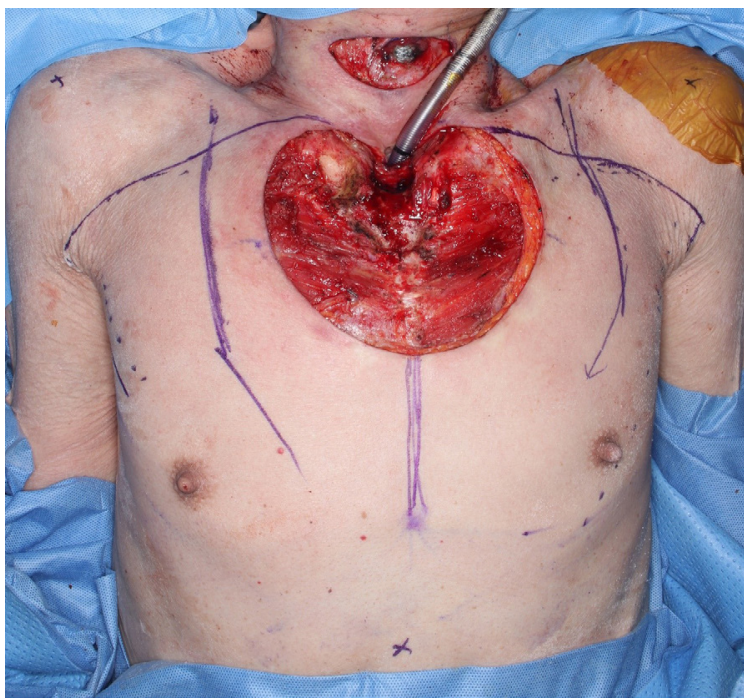

Fig. 2. Photograph showing a round soft-tissue defect on the upper mid-chest wall.

months before presentation, the tumor had gradually grown in the upper chest and neck, and the mass effect of the tumor on the trachea caused airway obstruction and severe dyspnea (Fig. 1). Incisional biopsy confirmed recurrent SCC, and a preoperative computed tomography scan revealed multiple small nodules in the lungs, suggesting metastasis of the primary cancer. We decided that conservative treatment to relieve dyspnea symptoms, given the rapidly growing nature of the mass, would be ineffective; therefore, palliative tumor resection was planned to improve symptoms. The chest mass was removed and a safety margin of about 1 or $2 \mathrm{~mm}$ was secured. The size of the remaining defect in the central area of the upper chest and sternum was

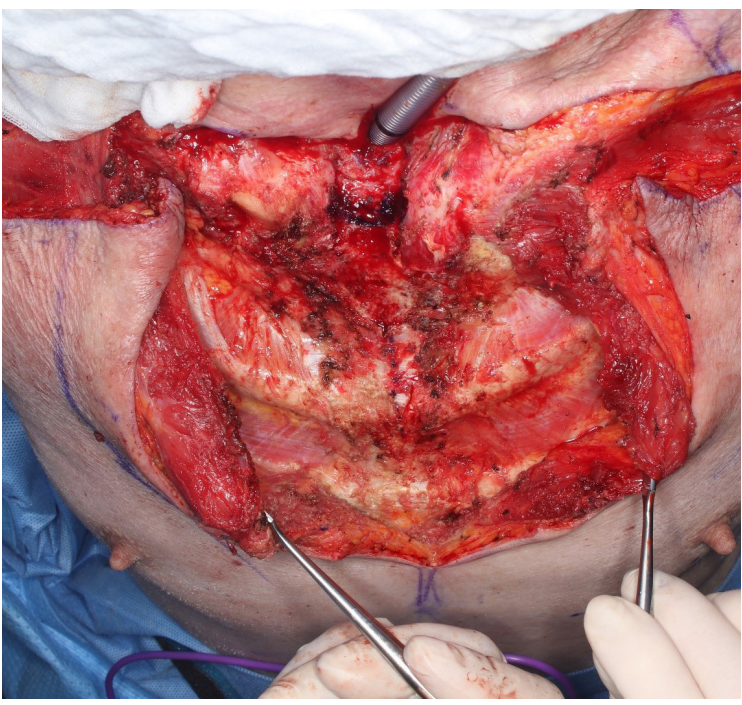

Fig. 3. The origins and insertions of the pectoralis major muscles were released.

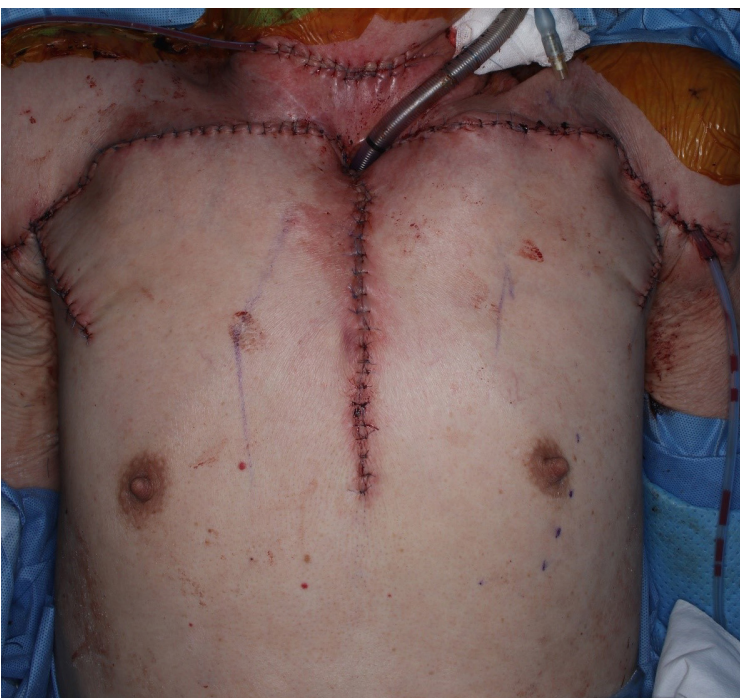

Fig. 4. Photograph showing the chest wall defect covered with bilateral pectoralis major myocutaneous and $\mathrm{V}-\mathrm{Y}$ rotation advancement flaps.

$10 \times 10 \mathrm{~cm}$ (Fig. 2). Reconstruction with bilateral PMMC flaps was planned after the margins had been determined to be negative by biopsy. The vertical height of the PMMC flaps, which were designed to be larger than the defect, was $15 \mathrm{~cm}$. Transversely, the flaps were designed up to the axillary area along the upper border of pectoralis major muscle. To enable elevation of the pectoralis muscle origins, anterior intercostal artery perforators were dissected by electrocautery, and after dissecting the pectoralis major muscles from the chest wall, PMMC flaps were elevated. However, they were insufficient to cover the defect. Therefore, to achieve further advancement, the clavicular and sternocostal heads of the pectoralis major muscles were dissect- 


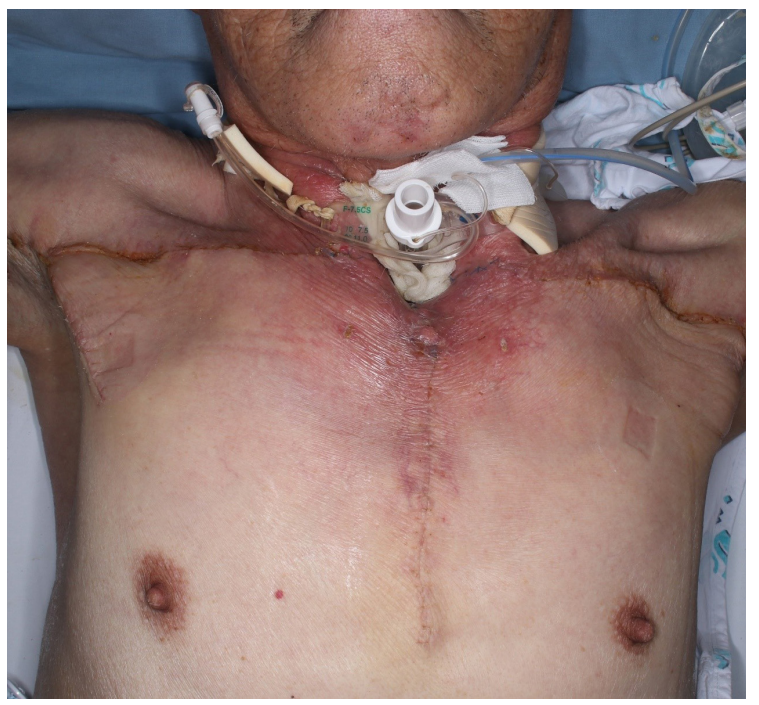

Fig. 5. Postoperative shoulder abduction.

ed and cut for further advancement; the muscles were cut $\sim 4 \mathrm{~cm}$ from their humeral insertion sites (Fig. 3). The pectoralis major muscle flaps were sufficient to cover the center of the chest, but skin movement was inadequate. Therefore, other procedures, such as additional skin grafts, were considered necessary. In this case, we opted for bilateral V-Y rotation advancement flaps for midline closure without an additional skin graft. After V-Y rotation advancement, skin was closed without tension (Fig. 4). The flaps remained viable and the patient recovered without any serious complications. At 4 weeks after surgery, no specific shoulder range of motion limitation was observed $\left(100^{\circ}\right.$ and $92^{\circ}$ of abduction on right and left sides, respectively) (Fig. 5). However, the patient died 9 months after surgery.

\section{DISCUSSION}

SCC of the head and neck is treated by surgery, radiation, and chemotherapy individually or in combination [5]. However, its recurrence rate is between $60 \%$ and $70 \%$ for local recurrence or regional metastasis, and between $20 \%$ and $30 \%$ for distant metastasis [6]. Lung metastases (66\%) are the most common form of distant metastases of SCC, followed by bone (22\%) and liver $(10 \%)$ metastases. Metastasis to the chest wall has been rarely reported [7]. Neoadjuvant or adjuvant radiotherapy is often performed for recurrent head and neck tumors, and 3 to 6 months after radiotherapy, reconstructive surgery should be performed carefully because the blood supply to tissues and fibroblast numbers decrease and delay wound healing [8]. During wide tumor resection, reconstructive surgeons should fill dead spaces in the chest cavity and prevent respiratory complications such as flail chest. For skeletal reconstruction, various types of prostheses and metal plates are used to secure skeletal structures [1-4,9]. With technical developments in microsurgery, free flaps have become the most important option for head and neck reconstruction. However, local flaps, including pectoralis major flaps are also indicated for patients with a poor general condition (i.e., when complete tumor resection cannot be performed). Although salvage operations are inevitable, free flaps are not indicated for these patients [10]. The reconstruction of soft tissue defects of the chest wall can be performed using local, PMMC, latissimus dorsi (LD), or vertical rectus abdominis muscle flaps depending on the defect size. LD musculocutaneous flaps can often be used to reconstruct anterior or anterolateral chest wall defects, but it is difficult to approach the mid-sternum area [2,3]. PMMC flaps such as unilateral PMMC, bilateral PMMC, and PMMC turn-over flaps are used to reconstruct mid-sternal defects $[2,3,11]$, and PMMC flaps based on the thoracoacromial pedicle are usually used as muscle advancement or rotation flaps. PMMC rotation flaps provide a useful way of addressing sternal defects, but if a defect is too large to cover with a unilateral PMMC flap, bilateral PMMC flaps may provide an appropriate option. Additionally, detachment of the sternocostal head from the clavicular head of the pectoralis major muscle or separation of the insertion site of the pectoralis major muscle aids flap advancement [3]. For larger defect coverage, a rectus abdominis flap or a free flap may be used. In the described case, reconstruction was attempted using PMMC flaps rather than a free flap, because the patient was an elderly cancer patient in poor general condition with recurrence. If a patient's general condition is favorable, a free flap or omental flap is also considered a good choice for very large defect coverage $[2,7,10]$. An axillary V-Y rotation advancement flap is a possible option for young patients, but is more appropriate for older patients with redundant skin, which is readily advanced, provides tension-free sutures, and does not cause severe postoperative shoulder motion restrictions. Unfortunately, there were no cases of young or female patients to refer to; therefore, we suggest studies be undertaken to determine the optimal treatment for such patients. We report this case treated using bilateral PMMC flaps, detaching the pectoralis major muscles from their origins and insertion sites, and using an axillary V-Y rotation advancement flap to maximize advancement after reconstruction of a large chest wall defect in an elderly patient without specific complications.

\section{NOTES}

\section{Conflict of interest}

Sae Hwi Ki is an editorial board member of the journal but was 
not involved in the peer reviewer selection, evaluation, or decision process of this article. No other potential conflicts of interest relevant to this article were reported.

\section{Ethical approval}

The study was performed in accordance with the principles of the Declaration of Helsinki.

\section{Patient consent}

The patient provided written informed consent for the publication and the use of his images.

\section{Author contribution}

Project administration: SH Ki. Writing - original draft: GY Jo, JM Yoon. Writing - review \& editing: SH Ki. All authors read and approved the final manuscript.

\section{ORCID}

Gang Yeon Jo

Jin Myung Yoon

Sae Hwi Ki

https://orcid.org/0000-0003-3951-9830

https://orcid.org/0000-0002-8129-0753

https://orcid.org/0000-0001-9194-9681

\section{REFERENCES}

1. Mansour KA, Thourani VH, Losken A, et al. Chest wall resections and reconstruction: a 25-year experience. Ann Thorac Surg 2002;73:1720-5.

2. Novoa NM, Alcaide JLA, Gomez Hernandez MT, et al. Chest wall - reconstruction: yesterday, today, and the future. Shanghai Chest 2019;3:15.
3. Bakri K, Mardini S, Evans KK, et al. Workhorse flaps in chest wall reconstruction: the pectoralis major, latissimus dorsi, and rectus abdominis flaps. Semin Plast Surg 2011;25:4354.

4. Weyant MJ, Bains MS, Venkatraman E, et al. Results of chest wall resection and reconstruction with and without rigid prosthesis. Ann Thorac Surg 2006;81:279-85.

5. Na SK, Cho HJ, Jeon JH, et al. The value of salvage operation for recurrent head and neck cancer after surgery alone or surgery with radiotherapy. Korean J Otolaryngol-Head Neck Surg 2001;44: 301-4.

6. Armand JP, Cvitkovic E, Recondo G, et al. Salvage chemotherapy in recurrent head and neck cancer: the Institut Gustave Roussy experience. Am J Otolaryngol 1993;14:301-6.

7. Ferlito A, Shaha AR, Silver CE, et al. Incidence and sites of distant metastases from head and neck cancer. ORL 2001; 63:202-7.

8. Haubner F, Ohmann E, Pohl F, et al. Wound healing after radiation therapy: review of the literature. Radiat Oncol 2012;7:162.

9. Turna A, Kavakli K, Sapmaz E, et al. Reconstruction with a patient-specific titanium implant after a wide anterior chest wall resection. Interact Cardiovasc Thorac Surg 2014;18: 234-6.

10. You YS, Chung CH, Chang YJ, et al. Analysis of 120 pectoralis major flaps for head and neck reconstruction. Arch Plast Surg 2012;39:522-7.

11. Solomon MP, Granick MS. Bipedicle muscle flaps in sternal wound repair. Plast Reconstr Surg 1998;101:356-60. 